\title{
Association Between Time to Stent Dysfunction and the Anti-Tumour Effect of Systemic Chemotherapy Following Stent Placement in Patients With Pancreaticobiliary Cancers and Malignant Gastric Outlet Obstruction: A Retrospective Cohort Study
}

Satoshi Kobayashi ( $\nabla$ kobayashis@kcch.jp )

Kanagawa Cancer Center

Makoto Ueno

Kanagawa Cancer Center

Shuhei Nagashima

Kanagawa Cancer Center

Yusuke Sano

Kanagawa Cancer Center

Kuniyuki Kawano

Kanagawa Cancer Center

Taito Fukushima

Kanagawa Cancer Center

Hiroyuki Asama

Kanagawa Cancer Center

Shun Tezuka

Kanagawa Cancer Center

Manabu Morimoto

Kanagawa Cancer Center

Research Article

Keywords: duodenal stenting, systemic chemotherapy, cancer, stent dysfunction, response

Posted Date: February 17th, 2021

DOl: https://doi.org/10.21203/rs.3.rs-199477/v1

License: (c) (1) This work is licensed under a Creative Commons Attribution 4.0 International License.

Read Full License 

1 Association between time to stent dysfunction and the anti-tumour effect of systemic

2 chemotherapy following stent placement in patients with pancreaticobiliary cancers and

3 malignant gastric outlet obstruction: A retrospective cohort study

5 Satoshi Kobayashi ${ }^{1}$, Makoto Ueno ${ }^{1}$, Shuhei Nagashima ${ }^{1}$, Yusuke Sano ${ }^{1}$, Kuniyuki Kawano ${ }^{1}$,

6 Taito Fukushima ${ }^{1}$, Hiroyuki Asama $^{1}$, Shun Tezuka $^{1}$, Manabu Morimoto $^{1}$

7

8 1. Department of Gastroenterology, Hepatobiliary and Pancreatic Medical Oncology

9 Division, Kanagawa Cancer Center

10 2-3-2, Nakao, Asahi-ku, Yokohama, 241-0815, Japan

\section{Corresponding author}

13 Satoshi Kobayashi

14 Complete postal address for correspondence/reprints

15 Department of Gastroenterology, Hepatobiliary and Pancreatic Medical Oncology Division,

16 Kanagawa Cancer Center

17 2-3-2, Nakao, Asahi-ku, Yokohama City, 241-0815, Japan

18 Tel: $+81-45-520-2222$

19 Fax: +81-45-520-2202 
20 Email: kobayashis@kcch.jp 


\section{Abstract}

23 Background: Malignant gastric outlet obstruction (MGOO) occasionally occurs due to

24 pancreaticobiliary cancer. Endoscopic duodenal stenting (DS) is a common treatment for

25 MGOO. However, it has been reported that DS does not have sufficient patency time for it to

26 be used in patients who have a potentially increased lifespan. Nowadays, systemic

27 chemotherapy for pancreaticobiliary cancer has developed, and its anti-tumour effect would make time to stent dysfunction longer.

Therefore, we retrospectively evaluated the association between objective response to systemic chemotherapy followed by DS and time to stent dysfunction in patients with advanced pancreaticobiliary cancer.

Methods: This retrospective study included 109 patients with advanced pancreaticobiliary cancer who received systemic chemotherapy after DS. Patients who showed complete or partial response were defined as responders. The rest were defined as non-responders. Time to stent dysfunction was compared between responders and non-responders using the landmark analysis, at 2 months after DS. Death without recurrence of MGOO was considered as a censored case for time to stent dysfunction.

Results: Combination and monotherapy regimens were adopted for 41 and 68 patients, respectively. Median progression-free survival and overall survival were 3.2 months (95\% 
41 observed in 21 patients $(19.3 \%)$. Patients who received combination regimens had longer

42 progression-free survival and higher response rates than those who received monotherapy

43 regimens; progression-free survival was 5.1 months (95\% CI, 3.1-7.0) and 2.6 months (95\%

44 CI, 1.6-3.5) with a p-value of $<0.001$, and response rates were $39.0 \%$ and $7.4 \%$ with a p-

value $<0.001$, respectively. Median time to stent dysfunction was 12.5 months $(95 \% \mathrm{CI}, 8.4-$

46 16.5) in the entire cohort. In 89 patients, responders had longer time to stent dysfunction than non-responders: 17.4 months (95\% CI, 17.3-17.5) and 7.1 months (95\% CI, 1.6-12.5), respectively, with a p-value of 0.031 .

Conclusions: Longer time to stent dysfunction is expected when systemic chemotherapy

following DS suppresses tumour progression; DS is slated to be a standard treatment for

MGOO even in patients with pancreaticobiliary cancer and a long lifespan.

Keywords: duodenal stenting, systemic chemotherapy, cancer, stent dysfunction, response

\section{BACKGROUND}

Pancreatic cancer is the third leading cause of cancer-related death in the United States; death due to this disease accounted for approximately 43,000 cases in 2017.[1] It was the fourth leading cause of cancer death in Japan in 2018.[2] Biliary tract cancer includes the malignant cancer originating from the extrahepatic and hilar bile ducts, gallbladder, and ampulla of Vater. 
61 Asian countries, accounting for 18,000 cases in Japan. As the disease progresses, the primary

62 tumour grow up; malignant gastric outlet obstruction (MGOO) due to tumour invasion through

63 the layers of the gastroduodenal wall occurs in approximately 10-20\% of patients with

64 pancreaticobiliary cancer.[3-5] Gastric outlet obstruction is a crucial issue for patients with

65 these cancers, because it induces nausea, vomiting, and anorexia, which can result in life-

66 threatening comorbidities.

67 Gastrojejunostomy (GJ) has been widely used as a palliative treatment option for MGOO; in

68 the early 1990s, duodenal stenting (DS) was first developed and reported as an alternative

69 procedure to GJ.[6] According to the reviews of some clinical trials, DS is especially

70 recommended for patients with MGOO who had a life expectancy of less than 3 months, since

71 the patency of DS is approximately 3 months and additional interventions are often

72 required.[7-9]

73 Advanced pancreaticobiliary cancer has a poor prognosis, even in patients who received

74 systemic chemotherapies after resolving MGOO, since systemic chemotherapies (such as gemcitabine (GEM) monotherapy) had little efficacy in prolonging patient survival and a low response rate.[10, 11] Patients with advanced pancreaticobiliary cancer were candidates for DS, even if they could receive subsequent chemotherapy after resolving MGOO. We evaluated the clinical effectiveness and safety of DS for patients with pancreatic cancer[12] and concluded that DS was an effective treatment for patients with advanced pancreatic cancer 
80 and MGOO, in terms of its safety and smooth performance of subsequent chemotherapies.

81 In recent years, systemic chemotherapies have been developed for advanced

82 pancreaticobiliary cancer, such as FOLFIRINOX (fluorouracil, leucovorin, irinotecan and

83 oxaliplatin)[13] and GEM plus nab-paclitaxel[14] for pancreatic cancer, and GEM plus

84 cisplatin[15] and GEM plus S-1 for biliary tract cancer.[16] Response rate for these regimens

85 has been reported as 30-60\%; overall survival was 10-11 months.[17] Based on these data,

86 patients who were candidates for these regimens will be suitable for GJ rather than for DS, as

87 the treatment option for MGOO; however, DS can be another option if chemotherapy can

88 prolong the patency of DS. Therefore, in this study, we aimed to assess the association

89 between time to stent dysfunction (TTSD) for DS and efficacy of systemic chemotherapy.

91 METHODS

$92 \quad$ Patients

93 This was a retrospective cohort study. We reviewed the medical records of 317 consecutive

94 patients with advanced pancreaticobiliary cancer who underwent DS for MGOO at our

95 institution between July 2010 and December 2019. Patients enrolled in this study were also

96 provided the opportunity to opt out of having any information published. 
99 We considered patients as candidates for endoscopic DS if they met the following criteria as

100 described in our previous report[12]: 1) unresectable or recurrent disease that could not be

101 cured with surgical resection; 2) histologically or cytologically proven pancreaticobiliary

cancer; and 3) MGOO due to a stricture in the stomach or duodenum that was confirmed

through radiological or endoscopic findings. Duodenal stent placement was contraindicated for

patients who met the following conditions: 1) small bowel strictures or functional disorder

induced by peritoneal dissemination; 2) stent placement risk factors due to haemorrhagic status

or cardiopulmonary problems; 3) life expectancy of less than two weeks. We used several types

of stents, such as the WallFlex or WallFlex Duodenal Soft (Boston Scientific Corporation,

Marlborough, MA, USA) (22 $\mathrm{mm}$ with a proximal flare of $24 \mathrm{~mm}$ in diameter); Niti-S D

Korea) (22 mm in diameter without proximal flares); HANAROSTENT® Naturfit ${ }^{\mathrm{TM}}$ Duo (22

$111 \mathrm{~mm}$ in diameter without proximal flares) (Boston Scientific Corporation, Marlborough, MA,

112 USA). Stent lengths vary from 60 to $120 \mathrm{~mm}$; we selected the stent according to the stricture

113 length. The Niti-S COMVI ${ }^{\mathrm{TM}}$ was only used for patients at risk of tumour haemorrhage because

114 it is a covered stent. Uncovered stents were used at the physician's discretion. Stent placement

115 was performed under sedation; the stricture was mainly identified endoscopically. In cases

116 where the stricture was located at the distal end of the horizontal portion of the duodenum, we 
118 Bayer HealthCare Pharmaceuticals, Leverkusen, Germany) to identify the stricture. A 0.035-

119 inch guidewire (Hydra Jagwire, Boston Scientific Corporation) was inserted through the

120 stricture, and the duodenal stent was positioned across it, under fluoroscopic guidance. Stent

121 length was chosen according to the stricture length and the position of the pylorus/ampulla of

122 Vater. Finally, the stent was deployed, and its patency was confirmed by injection of the

123 contrast medium. Types of DSs used with each patient depended on the physician's discretion

124 and their availability. After stent placement, patients could resume consuming liquids on day

1251 , soft foods on day 2, and solid foods on day 3, as long as no symptoms of MGOO exacerbation

126 were observed. In cases of MGOO recurrence, a second/third duodenal stent was placed using

127 the stent-in-stent technique.

129 Systemic chemotherapy after duodenal stenting

130 We considered applying systemic chemotherapy after duodenal stenting for patients who met

131 the following criteria: 1) Eastern Cooperative Oncology Group performance status (ECOG PS)

132 of $0-2 ; 2$ ) achievement of full oral intake; 3) adequate liver and kidney functions; 4) preserved

133 bone marrow function: neutrophil count $>1500 / \mu \mathrm{L}$; platelet count $>100,000 / \mu \mathrm{L}$; and 5) a life

134 expectancy of at least 3 months. We chose the chemotherapy regimens according to each

135 patient's consent and conditions, as follows: e.g., patients who had ECOG PS of 0-1 and were aged $<75$ years were introduced to combination chemotherapeutic regimens, such as modified 
137 FOLFIRINOX[18] or GEM plus nab-paclitaxel for pancreatic cancer, or GEM plus cisplatin

138 or GEM plus S-1 for biliary tract cancer. Patients who were ineligible for these combination

139 regimens (but eligible for less toxic therapies) were introduced to monotherapy regimens, such

140 as GEM alone or S-1 alone. We adopted these regimens not only as first-, but also as second-

141 or third-line treatments. The initial dose of each agent was reduced at the physician's discretion.

142 Chemotherapy continued until disease progression, intolerable adverse events, or patient 143 refusal.

\section{Clinical outcomes}

146 Diagnosis of stent occlusion or migration was confirmed based on radiological and/or

147 endoscopic findings and not on symptoms such as nausea or vomiting. TTSD was determined

148 starting from the date of DS through the date of diagnosis of stent dysfunction. Tumour

149 ingrowth and overgrowth, stent migration, and food impaction were considered as stent

150 dysfunction-related events. Patients who died without stent dysfunction were treated as

151 censored cases for the evaluation of TTSD. Additionally, we complimentarily evaluated TTSD

152 by considering the impossibility of oral intake due to disease progression. Overall survival and

153 progression-free survival were determined starting on the date of initiation of systemic

154 chemotherapy through the date of documented disease progression or any cause of death and

155 the date of death due to any cause or the last follow-up, respectively. The best response during 
156 chemotherapy was radiologically evaluated according to the Response Evaluation Criteria in

157 Solid Tumors version 1.1.[19]

159 Statistical analysis

160 Results are expressed as median and were analysed using the SPSS Statistics 23 software (IBM

161 SPSS, Inc., Chicago, IL, USA). Changes in the MGOO score[4] before and after duodenal stent

162 placement were evaluated using the Wilcoxon signed-rank test. We calculated overall survival,

163 progression-free survival, and TTSD using the Kaplan-Meier method; we used the log-rank

164 test to compare these time-to-event parameters in two groups. Objective response to systemic

165 chemotherapy was evaluated according to the new response evaluation criteria in solid tumours,

166 version 1.1.[19] Patients were divided into the two groups according to the objective response

167 to chemotherapy. Patients who showed complete or partial responses were defined as

168 responders, and those who showed stable or progressive disease were defined as non-

169 responders. We compared the TTSD of responders with that of non-responders by conducting

170 a landmark analysis (landmark at 2 months after DS) to avoid guarantee-time bias and evaluate

171 the association between TTSD and efficacy of systemic chemotherapy after DS.

173 RESULTS

174 Patient characteristics 
175 Of 319 consecutive patients who had undergone DS, the study included 109 patients who

176 received systemic chemotherapy after DS (Figure 1). Patient characteristics are shown in Table

177 1. Primary diseases were pancreatic adenocarcinoma, biliary tract cancer, and pancreatic neuroendocrine neoplasm in 92, 12, and 3 patients, respectively. Median age of the enrolled patients was 68 years (range: $31-81$ ); 85 patients (77\%) had metastatic cancer and 52 of them had peritoneal dissemination.

\section{Duodenal stent placement}

183 The duodenal stents used were WallFlex, Niti-S D type, HANAROSTENT ${ }^{\circledR}$ Naturfit ${ }^{\text {TM }}$ Duo,

184 Niti-S COMVI ${ }^{\mathrm{TM}}$, and WallFlex Soft in 46, 46, 10, 4, and 3 patients, respectively. Strictures were located on the oral side, above, and anal side of the papilla of Vater in 33, 26 and 50 patients, respectively. Among those, 6 patients with a MGOO score of 3 required DS: MGOO on the horizontal part of the duodenum was complicated with cholangitis in 3 patients; 2 patients had strictures in both the duodenal bulb and common bile duct; hence, DS was required before endoscopic retrograde biliary stenting. The MGOO score was better after DS compared to that before it, with a p-value $<0.001$. The median time required to tolerate food intake was just 1 day (range: 1-18 days). 
194 Median time from the date of stent placement through the date of initiation of chemotherapy

195 was 12 (range: 1-60) days. Combination and monotherapy regimens were adopted for 41 and

19668 patients, respectively. Median progression-free survival and overall survival were 3.2

197 months (95\% CI, 2.4-4.0) and 6.0 months (95\% CI, 4.6-7.3) (Figures 2a and 2b). Regarding

198 the objective response, partial response (PR), stable disease (SD), and progressive disease (PD)

199 were observed in 21, 45, and 43 patients, respectively. Response and disease control rates were

$20019.2 \%$ and $60.6 \%$, respectively. Patients who received combination regimens had longer

201 progression-free survival and higher response rates than those who received monotherapy

202 regimens; progression-free survival was 5.1 months (95\% CI, 3.1-7.0) and 2.6 months (95\%

203 CI, 1.6-3.5) with a p-value of $<0.001$ (Figure 2c), and response rates were $39.0 \%$ and $7.4 \%$ with

204 a p-value $<0.001$, respectively. In addition, 17 patients who received combination regimens as

205 first-line treatments had a median progression-free survival of 8.6 months (95\% CI, 1.5-15.6)

206 (Supplement Figure 1) and a response rate of 58.8\%.

207

208

\section{Time to duodenal stent dysfunction}

209 During the median observation time of 4.8 (range: $0.5-35.6$ ) months, stent dysfunction was

210 observed in 38 patients (35\%) and impossibility of oral intake due to disease progression was

211 observed in 53 (49\%); the remaining 19 patients were censored at the time of analysis. The

212 median TTSD was 12.5 months (95\% CI, 8.4-16.5) (Figure 3a). When using a supplementary 
213 definition of stent dysfunction, median TTSD was 5.0 months (95\% CI, 4.3-5.7) in the entire

214 cohort and 12.5 months (95\% CI, 2.7-22.3) in patients who received combination regimens as

215 the first-line treatment (Supplement Figure 2).

216 Landmark analysis at the 2-month landmark point revealed that TTSD was longer in responders

$217(\mathrm{n}=21)$ than in non-responders $(\mathrm{n}=68),[17.4$ months $(95 \%$ CI, 17.3-17.5) and 7.1 months

218 (95\% CI, 1.6-12.5) with a p-value of 0.031, respectively] (Figure 3b). There were differences

219 in TTSD between responders and non-responders in patients who received combination

220 regimen (Figure 3c) or a first-line treatment after DS (Figure 3d), with p-values of 0.043 and

221 0.024, respectively. Multivariable analysis showed that non-responders was a marginal poor

222 prognostic factor for TTSD; hazard ratio was 2.5 (95\% CI, 0.93-6.7), with a p-value of 0.070

223 (Table 2).

224

225

226 DISCUSSION

227 According to previous studies, DS is inferior to GJ in terms of TTSD, although DS is superior

228 to GJ in terms of safety, rapid symptom relief, and shorter time required to resume food intake.

229 Based on the results, treatment recommendations for pancreatic cancer by the National

230 Comprehensive Cancer Network and Japanese Pancreas Society state that proper use of the

231 two treatment options may depend on the patients' prognostic estimates.[20][21] These 
232 recommendations do not state which is better for patients who will receive chemotherapy; their

233 references date from 2000 to 2010 , when the response rate of systemic chemotherapy was

234 dismal. In recent decades, systemic chemotherapy for advanced pancreaticobiliary cancer has

235 developed. Therefore, we evaluated the influence of chemotherapy on TTSD in patients who

236 underwent DS and subsequent chemotherapy in 2010-2018. Our results showed that TTSD

237 after DS was prolonged after chemotherapy through tumour shrinkage, especially when using

238 a combination regimen. Therefore, DS could be a good treatment option, not only for patients

239 with short life expectancy, but also for those who are eligible for a combination regimen and

240 can expect an increased life expectancy. In other words, DS can be applied to all patients with

241 unresectable pancreaticobiliary cancer as a standard treatment option for MGOO, along with

242 advances in chemotherapy.

243 There are several reports on the effect of chemotherapy on TTSD of biliary stent; some studies

244 have concluded that chemotherapy decreased TTSD by inducing bacterial colonisation in the

245 bile ducts through its side effect of immunosuppression;[22] others have stated opposite

246 conclusions, speculating that positive effect via tumour control would compensate for its

247 negative effect in patients who received chemotherapy.[23] Regarding DS, the main causes of

248 stent dysfunction are tumour growth and food impaction. Bacterial colonisation does not

249 influence its patency. Therefore, we believe this might be a reason why chemotherapy has

positive effects on TTSD of DS. Kim et al. have also reported that time to progression was an 
251 independent protective factor against restenosis of DS in patients with gastric cancer.[24] When

252 that report was published, the response rate and time to progression of chemotherapy for

253 unresectable gastric cancer was around 30-40\% and 5-7 months, respectively.[25, 26] These

254 values were close to those of recent standard chemotherapy for advanced pancreaticobiliary

255 cancer.[13-16] Patients who received combination regimens in our study had a median

256 progression-free survival of 5.1 months and response rate of $39.0 \%$. Therefore, we think this

257 was another reason why our study could show the association between TTSD for DS and the

258 effects of chemotherapy.

259 Overall survival was 4.6 months or longer in $95 \%$ of the patients included in our cohort.

260 According to this review article,[7] surgical bypass is recommended for patients with a life

261 expectancy of 3 months or longer; most patients had been eligible for surgical bypass rather

262 than for DS. However, in this study, TTSD for DS was long enough that the patients survived

263 without recurrent MGOO; median TTSD was 12.5 months in the primary definition of stent

264 dysfunction and median time to impossibility of oral intake was 5.0 months. Furthermore,

265 patients who received combination regimens as the first-line treatment could have 12.5 months

266 of oral food intake. Based on the results, we believe that DS could be also recommended as a

267 treatment option for MGOO for patients who are scheduled to receive intensive first-line

268 chemotherapy for unresectable pancreaticobiliary cancer. Development of systemic

269 chemotherapy for these diseases is awaited; DS will have longer TTSD in the future than 
270 nowadays along with the improvement in response rate and progression-free survival in the

271 future. Regarding safety, DS is considered a better option than surgical bypass, unless

272 complications included stent dysfunction.[7,9] Although we did not compare DS with surgical

273 bypass in this study, we believe that DS is neither inferior to surgical bypass in terms of efficacy,

274 nor superior in terms of safety.

275 The existing definition of stent dysfunction has affected the results, as the majority of patients

276 could not eat orally due to disease progression; their stents were found to be patent, even in the

277 final phase of the life. Regarding biliary stenting, the TOKYO criteria (2014) for transpapillary

278 biliary stenting recommend patient death and complications other than recurrent biliary

279 obstruction requiring stent removal be treated as censored cases at the time of death or stent

280 removal, respectively.[27] According to the above-mentioned criteria, our primary definition

281 of stent dysfunction was set as recurrent MGOO with stent thrombosis or migration. Primary

282 data analysis showed that stent dysfunction was only observed in one third of patients. The

283 small number of events will lower the statistical power to detect differences; hence, we

284 conducted a supplementary analysis using disease progression as an event, to support the

285 robustness of the primary analysis.

286 This study has some limitations. Its small number of patients and retrospective design are likely

287 to cause several biases. Our study did not have a surgical bypass cohort; therefore, direct comparison between DS and surgical bypass was impossible. Indications for each 
289 chemotherapy regimen depended on the physician's discretion. In addition, patients and

290 regimens in this study formed a complex combination of several diseases and regimens.

\section{CONCLUSIONS}

293 Despite these limitations, we concluded that the anti-tumour effects of systemic chemotherapy

294 improve TTSD of DS in pancreaticobiliary cancers; DS could be preferable not only for

295 patients who have a life expectancy of less than 3 months, but also for those who are scheduled

296 to receive systemic chemotherapy and are slated to have a longer life expectancy. Therefore,

297 DS can be the standard treatment option for MGOO in unresectable pancreaticobiliary cancers.

298

299

$300 \quad$ List of Abbreviations

301 MGOO, malignant gastric outlet obstruction

302 DS, duodenal stenting

303 TTSD, time to stent dysfunction

304 ECOG PS, Eastern Cooperative Oncology Group performance status 


\section{DECLARATIONS}

Ethics approval and consent to participate

310 All patients provided written informed consent for duodenal stent placement; all eligible patients for subsequent chemotherapy also provided written informed consent. In addition, this

312 study was performed in accordance with the Declaration of Helsinki, and approved by the

313 Institutional Review Board of the Kanagawa Cancer Center in April 2020.

\section{Consent for publication}

316 Not applicable

\section{Availability of data and materials}

319 The datasets analysed during the current study are available from the corresponding author on reasonable request.

\section{Competing Interests}

S.K received honoraria from Boston Scientific and Taiho Pharmaceutical. M.U. received honoraria from MSD, Ono Pharmaceutical, Taiho Pharmaceutical, and Yakult Honsha Co., Ltd.

All other authors have no conflict of interest to declare. 
Funding

This was a self-funded study.

\section{Authors' contributions}

SK, SN, YS, KK, TF, HA, and ST analysed the patient data regarding the efficacy of duodenal

\section{Acknowledgements}

None

\section{References}

1. Siegel RL, Miller KD, Jemal A: Cancer statistics, 2018. CA: a cancer journal for clinicians 2018, 68(1):7-30.

2. https://www.e-stat.go.jp/en/stat-search?page $=1 \&$ toukei=00450011\&bunya_l=02 Accessed Feb 07, 2020

3. Espinel J, Vivas S, Munoz F, Jorquera F, Olcoz JL: Palliative treatment of malignant obstruction of gastric outlet using an endoscopically placed enteral Wallstent. Digestive diseases and sciences 2001;46:2322-2324.

4. Adler DG, Baron TH: Endoscopic palliation of malignant gastric outlet obstruction using selfexpanding metal stents: experience in 36 patients. The American journal of gastroenterology 2002;97:72-78.

5. Kulke MH: Metastatic pancreatic cancer. Curr Treat Options Oncol 2002;3:449-457.

6. Topazian M, Ring E, Grendell J: Palliation of obstructing gastric cancer with steel mesh, selfexpanding endoprostheses. Gastrointestinal endoscopy 1992;38:58-60. 
7. Jeurnink SM, van Eijck CH, Steyerberg EW, Kuipers EJ, Siersema PD: Stent versus gastrojejunostomy for the palliation of gastric outlet obstruction: a systematic review. $B M C$ gastroenterology 2007;7:18.

8. Jeurnink SM, Steyerberg EW, van Hooft JE, van Eijck CH, Schwartz MP, Vleggaar FP, Kuipers EJ, Siersema PD, Dutch SSG: Surgical gastrojejunostomy or endoscopic stent placement for the palliation of malignant gastric outlet obstruction (SUSTENT study): a multicenter randomized trial. Gastrointestinal endoscopy 2010;71:490-499.

9. Nagaraja V, Eslick GD, Cox MR: Endoscopic stenting versus operative gastrojejunostomy for malignant gastric outlet obstruction-a systematic review and meta-analysis of randomized and non-randomized trials. Journal of gastrointestinal oncology 2014;5:92-98.

10. Burris HA, 3rd, Moore MJ, Andersen J, Green MR, Rothenberg ML, Modiano MR, Cripps MC, Portenoy RK, Storniolo AM, Tarassoff $\mathrm{P}$ et al: Improvements in survival and clinical benefit with gemcitabine as first-line therapy for patients with advanced pancreas cancer: a randomized trial. Journal of clinical oncology : official journal of the American Society of Clinical Oncology 1997;15:2403-2413.

11. Suzuki E, Furuse J, Ikeda M, Okusaka T, Nakachi K, Mitsunaga S, Ueno H, Morizane C, Kondo S, Shimizu S et al: Treatment efficacy/safety and prognostic factors in patients with advanced biliary tract cancer receiving gemcitabine monotherapy: an analysis of 100 cases. Oncology 2010;79:39-45.

12. Kobayashi S, Ueno M, Kameda R, Moriya S, Irie K, Goda Y, Tezuka S, Yanagida N, Ohkawa S, Aoyama $\mathrm{T}$ et al: Duodenal stenting followed by systemic chemotherapy for patients with pancreatic cancer and gastric outlet obstruction. Pancreatology : official journal of the International Association of Pancreatology 2016;16:1085-1091.

13. Conroy T, Desseigne F, Ychou M, Bouche O, Guimbaud R, Becouarn Y, Adenis A, Raoul JL, Gourgou-Bourgade S, de la Fouchardiere $\mathrm{C}$ et al: FOLFIRINOX versus gemcitabine for metastatic pancreatic cancer. The New England journal of medicine 2011;364:1817-1825.

14. Von Hoff DD, Ervin T, Arena FP, Chiorean EG, Infante J, Moore M, Seay T, Tjulandin SA, Ma WW, Saleh MN et al: Increased survival in pancreatic cancer with nab-paclitaxel plus gemcitabine. The New England journal of medicine 2013;369:1691-1703.

15. Valle J, Wasan H, Palmer DH, Cunningham D, Anthoney A, Maraveyas A, Madhusudan S, Iveson T, Hughes S, Pereira SP et al: Cisplatin plus gemcitabine versus gemcitabine for biliary tract cancer. The New England journal of medicine 2010;362:1273-1281.

16. Morizane C, Okusaka T, Mizusawa J, Katayama H, Ueno M, Ikeda M, Ozaka M, Okano N, Sugimori K, Fukutomi A et al: Combination gemcitabine plus S-1 versus gemcitabine plus cisplatin for advanced/recurrent biliary tract cancer: the FUGA-BT (JCOG1113) randomized phase III clinical trial. Annals of Oncology 2019;30:1950-1958.

17. Ueno H, Ikeda M, Ueno M, Mizuno N, Ioka T, Omuro Y, Nakajima TE, Furuse J: Phase I/II study of nab-paclitaxel plus gemcitabine for chemotherapy-naive Japanese patients with 
metastatic pancreatic cancer. Cancer chemotherapy and pharmacology 2016;77:595-603.

18. Ozaka M, Ishii H, Sato T, Ueno M, Ikeda M, Uesugi K, Sata N, Miyashita K, Mizuno N, Tsuji K et al: A phase II study of modified FOLFIRINOX for chemotherapy-naive patients with metastatic pancreatic cancer. Cancer chemotherapy and pharmacology 2018; 81:1017-1023.

19. Eisenhauer EA, Therasse P, Bogaerts J, Schwartz LH, Sargent D, Ford R, Dancey J, Arbuck S, Gwyther S, Mooney M et al: New response evaluation criteria in solid tumours: revised RECIST guideline (version 1.1). European journal of cancer 2009;45:228-247.

20. Japanese Society of Pancreas, Treatment Guideline of Pancreatic Cancer. Kanehara Shuppan, 2019

21. National Comprehensive Cancer Network Guideline for Pancreatic Adenocarcinoma Version 1. 2020. https://www.nccn.org/professionals/physician_gls/pdf/pancreatic.pdf. accessed on 2 Mar 2020.

22. Lofts FJ, Evans TR, Mansi JL, Glees JP, Knight MJ: Bile duct stents: is there an increased rate of complications in patients receiving chemotherapy? European journal of cancer 1997; 33:209213.

23. Haal S, van Hooft JE, Rauws EAJ, Fockens P, Voermans RP: Stent patency in patients with distal malignant biliary obstruction receiving chemo(radio)therapy. Endoscopy international open 2017;5:E1035-e1043.

24. Kim CG, Park SR, Choi IJ, Lee JY, Cho SJ, Park YI, Nam BH, Kim YW: Effect of chemotherapy on the outcome of self-expandable metallic stents in gastric cancer patients with malignant outlet obstruction. Endoscopy 2012;44:807-812.

25. Ajani JA, Rodriguez W, Bodoky G, Moiseyenko V, Lichinitser M, Gorbunova V, Vynnychenko I, Garin A, Lang I, Falcon S: Multicenter phase III comparison of cisplatin/S-1 with cisplatin/infusional fluorouracil in advanced gastric or gastroesophageal adenocarcinoma study: the FLAGS trial. Journal of clinical oncology : official journal of the American Society of Clinical Oncology 2010;28:1547-1553.

26. Bang YJ, Van Cutsem E, Feyereislova A, Chung HC, Shen L, Sawaki A, Lordick F, Ohtsu A, Omuro Y, Satoh T et al: Trastuzumab in combination with chemotherapy versus chemotherapy alone for treatment of HER2-positive advanced gastric or gastro-oesophageal junction cancer (ToGA): a phase 3, open-label, randomised controlled trial. Lancet 2010;376:687-697.

27. Isayama H, Hamada T, Yasuda I, Itoi T, Ryozawa S, Nakai Y, Kogure H, Koike K: TOKYO criteria 2014 for transpapillary biliary stenting. Digestive endoscopy : official journal of the Japan Gastroenterological Endoscopy Society 2015;27:259-264. 
427 Figure 1 . CONSORT flow diagram of the study

428 Of 319 patients who received duodenal stent placement for malignant gastric outlet 429 obstruction due to advanced pancreaticobiliary cancer, 109 received systemic chemotherapy 430 followed by duodenal stent placement, and 89 had two months or longer to stent dysfunction.

432 Figure 2.

433 Kaplan-Meier curve of (a) overall survival and (b) progression-free survival

434 In the entire cohort, which consisted of 109 patients, median progression-free survival and 435 overall survival rates after duodenal stent placement were 3.2 months ( $95 \%$ confidence interval, 436 2.4-4.0) and 6.0 months (95\% confidence interval, 4.6-7.3), respectively.

437 (c) Comparison of 41 patients who received combination regimen (solid line) and 68 patients 438 who received a monotherapy regimen (dotted line), median progression-free survival rates 439 were 5.1 months (95\% confidence interval, 3.1-7.0) and 2.6 months (95\% confidence interval, $440 \quad 1.6-3.5)$ respectively, with a p-value of $<0.001$

442 Figure 3. Time to stent dysfunction

443 (a) Entire cohort

444 Median time to stent dysfunction was 12.5 months (95\% confidence interval, 8.4-16.5);

(b) Comparison between patients who showed a response (solid line) and those who did not 
(dotted line).

447 Median time from the 2-month landmark point to stent dysfunction was 17.4 months (95\% confidence interval, 17.3-17.5) in patients who showed a response and 7.1 months $(95 \%$ confidence interval, 1.6-12.5) in those who did not, with a p-value of 0.031 ;

(c) Comparison between responders and non-responders among patients who received combination regimen.

Supplement Figure 1. Kaplan-Meier curves of progression-free survival in patients who

(d) Comparison between responders and non-responders among patients who received systemic chemotherapy after duodenal stent placement as the first-line chemotherapy.

Median time from the 2-month landmark point to stent dysfunction was 33.6 months (95\% confidence interval, non-evaluable) in responders and 5.6 months (95\% confidence interval, non-evaluable) in non-responders, with a p-value of 0.024 . received a combination regimen as the first- (solid line), second-, or third-line line treatments (broken line) and those who received monotherapy as the first- (chained line), second-, or third- 
465 line treatments (dotted line).

466 Median progression-free survival with 95\% confidence interval was 5.8 months (0.2-11.5), 3.6

467 months (2.4-4.9), 1.1 months (0.2-2.0) and 2.8 months (0.9-4.6), respectively.

468

469 Supplement Figure 2. Kaplan-Meier curve of time to stent dysfunction when impossible oral

470 food intake was considered an event of stent dysfunction.

471 Median time to stent dysfunction was 5.0 months (95\% CI, 4.3-5.7) in the entire cohort (solid

472 line) and 12.5 months (95\% CI, 2.7-22.3) in patients who received combination regimens as

473 the first-line treatment (dotted line). 


\section{TABLES}

476 Table 1. Baseline characteristics of patients at initiation of systemic chemotherapy following

477 duodenal stent placement

\section{Characteristics}

Age (years), median (median)

$68.0(31-81)$

Sex, n (\%)

Male

$54(50)$

Female

$55(50)$

Primary disease, n (\%)

Pancreatic adenocarcinoma

$90(83)$

Biliary tract adenocarcinoma

$15(14)$

Pancreatic neuroendocrine tumour

$4(4)$

Disease status, $\mathrm{n}(\%)$

Locally advanced

$24(22)$

Metastatic

$85(78)$

ECOG performance status

0

$18(17)$

1

$62(57)$

2

$29(27)$

Position of bowel stricture, n (\%)

Oral side of the papilla of Vater

$33(30)$

Above the papilla of Vater

$26(24)$

Anal side of the papilla of Vater

$50(46)$ 


\begin{tabular}{|c|c|}
\hline \multicolumn{2}{|l|}{ Duodenal stent, n (\%) } \\
\hline WallFlex Duodenal & $46(42)$ \\
\hline WallFlex Duodenal Soft & $3(3)$ \\
\hline Niti-S D type & $46(42)$ \\
\hline Niti-S COMVI ${ }^{\mathrm{TM}}$ & $4(4)$ \\
\hline HANAROSTENT® Naturfit ${ }^{\mathrm{TM}}$ Duo & $10(9)$ \\
\hline \multicolumn{2}{|c|}{$\begin{array}{l}\text { Number of chemotherapy regimens prior to duodenal stenting, } \mathrm{n} \\
\text { (\%) }\end{array}$} \\
\hline 0 & $47(43)$ \\
\hline 1 & $49(45)$ \\
\hline $2-3$ & $13(12)$ \\
\hline CRP (mg/dL), median (range) & $0.79(0.05-12.9)$ \\
\hline \multicolumn{2}{|l|}{ Distribution, n (\%) } \\
\hline$<1.0 \mathrm{mg} / \mathrm{dL}$ & $60(55)$ \\
\hline$\geq 1.0 \mathrm{mg} / \mathrm{dL}$ & $49(45)$ \\
\hline Albumin (g/dL), median (range) & $3.3(2.1-4.1)$ \\
\hline \multicolumn{2}{|l|}{ Distribution, $\mathrm{n}(\%)$} \\
\hline$<3.5 \mathrm{mg} / \mathrm{dL}$ & $67(62)$ \\
\hline$\geq 3.5 \mathrm{mg} / \mathrm{dL}$ & $42(39)$ \\
\hline CA19-9 (U/mL), median (range) & $928.9(0-408800.0)$ \\
\hline \multicolumn{2}{|l|}{ Distribution, n (\%) } \\
\hline$<1,000 \mathrm{U} / \mathrm{mL}$ & $55(50)$ \\
\hline$\geq 1,000 \mathrm{U} / \mathrm{mL}$ & $54(50)$ \\
\hline
\end{tabular}


479 carbohydrate antigen 19-9

480 
481 Table 2. Multivariate analysis of prognostic factor for time to stent dysfunction ${ }^{\dagger}$

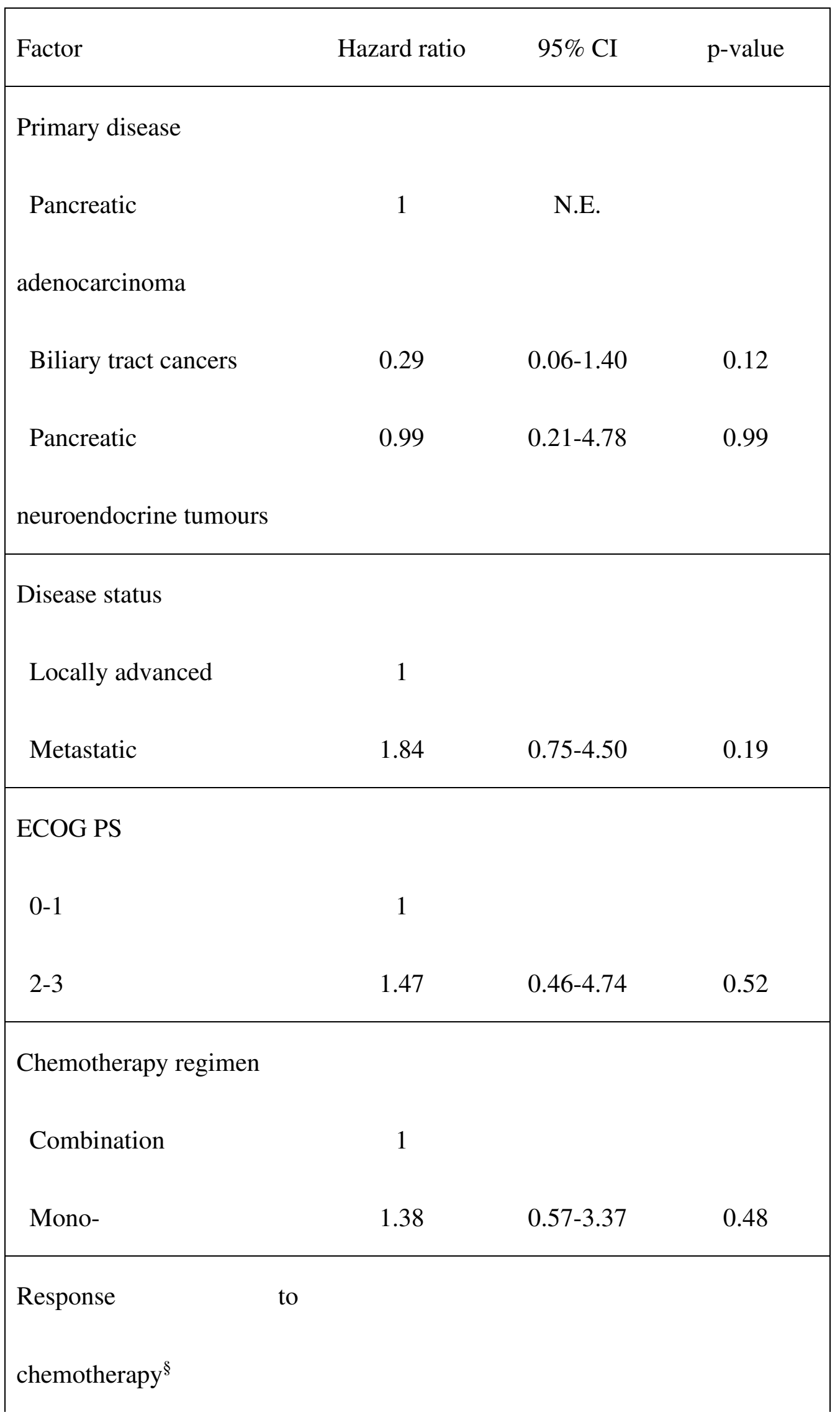




\begin{tabular}{|lccc|} 
Partial response & 1 & & \\
Stable or progressive & 2.49 & $0.93-6.67$ & 0.070 \\
disease & & & \\
\hline
\end{tabular}

$482 \uparrow$ Cox proportional hazards regression model in patients who had a 2-month or longer time to 483 stent dysfunction

$484 \S$ According to the New response evaluation criteria in solid tumours, version 1.1

4851 CI, confidence interval; ECOG PS, Eastern Cooperative Oncology Group performance 486 status; Combo, combination regimens such as FOLFIRINOX, gemcitabine plus nab-paclitaxel, 487 gemcitabine plus cisplatin and gemcitabine plus S-1; Mono, monotherapy such as gemcitabine 488 alone or S-1 alone 489 490 491 


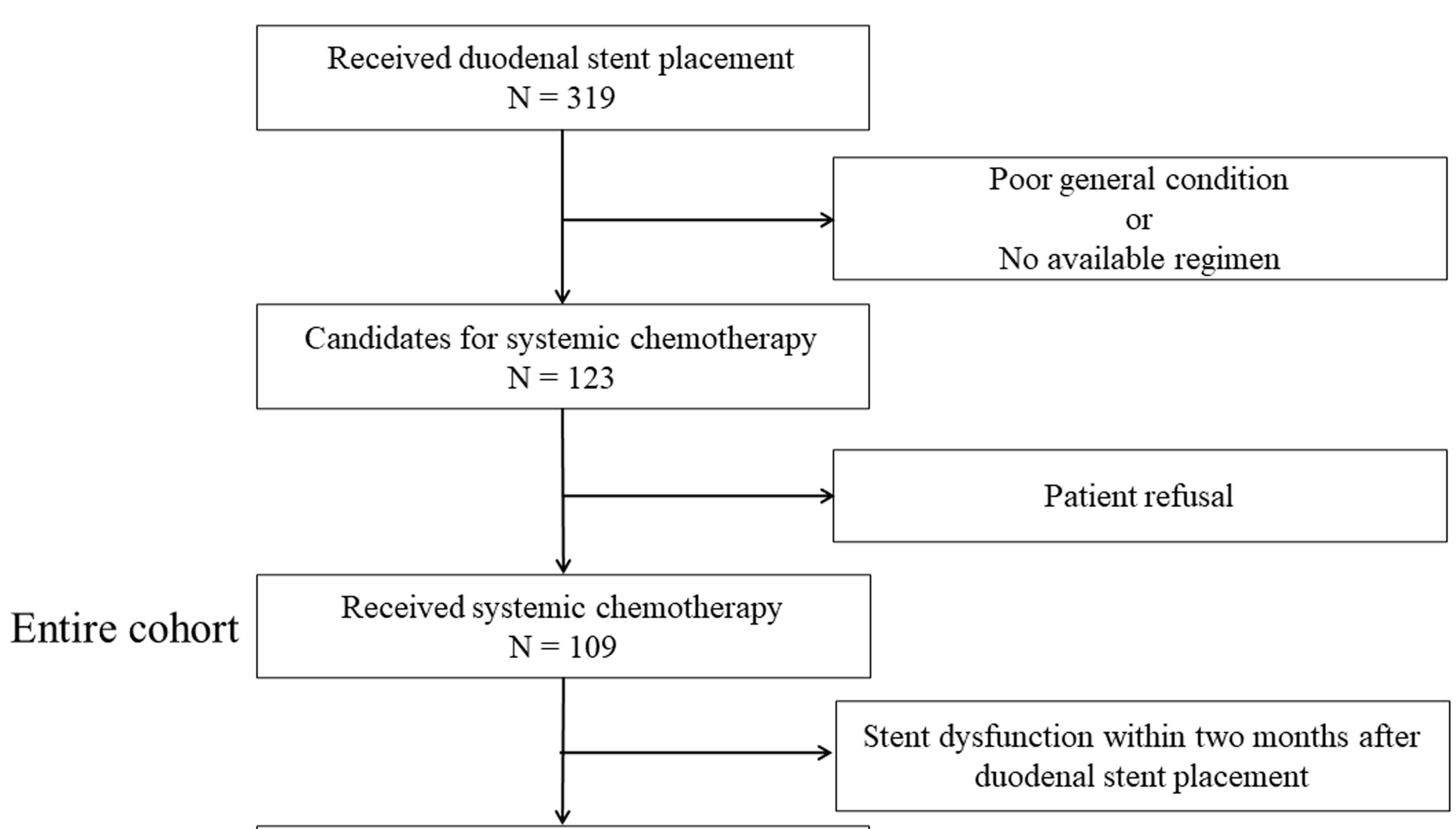

Object of landmark analysis

Two months or longer stent patency

$\mathrm{N}=89$

\section{Figure 1}



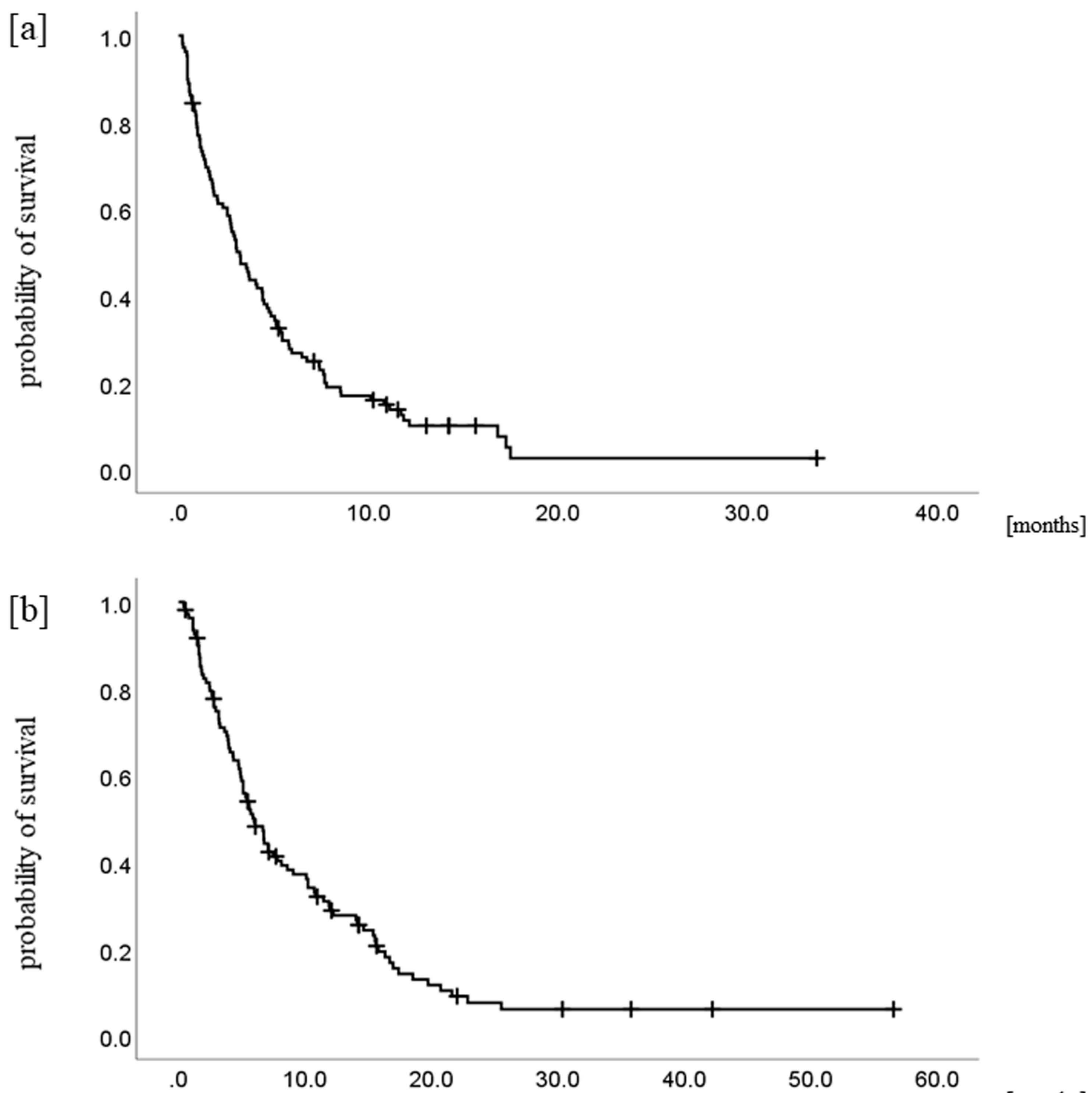

[months]

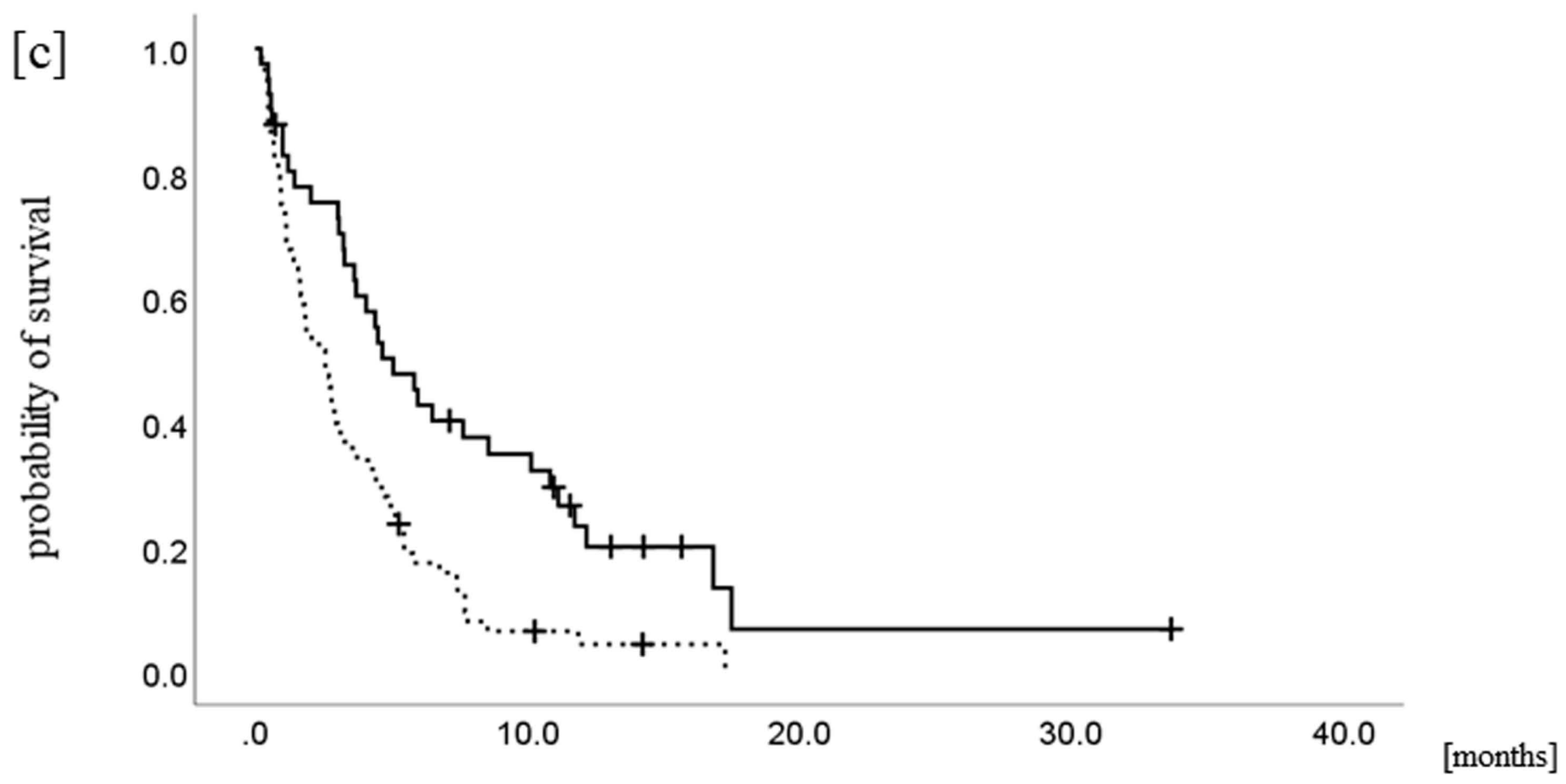

Figure 2 

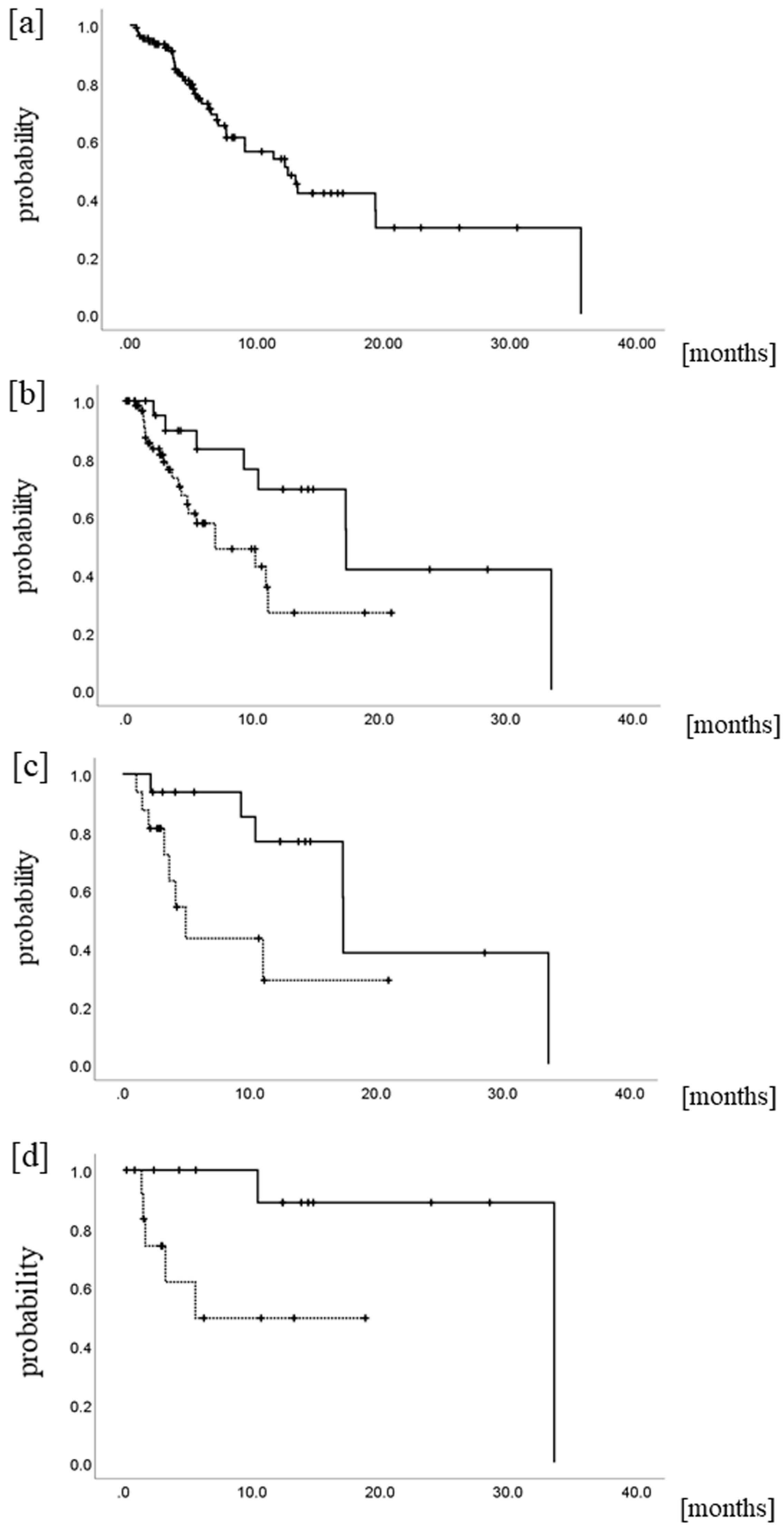

Figure 3 


\section{Figures}

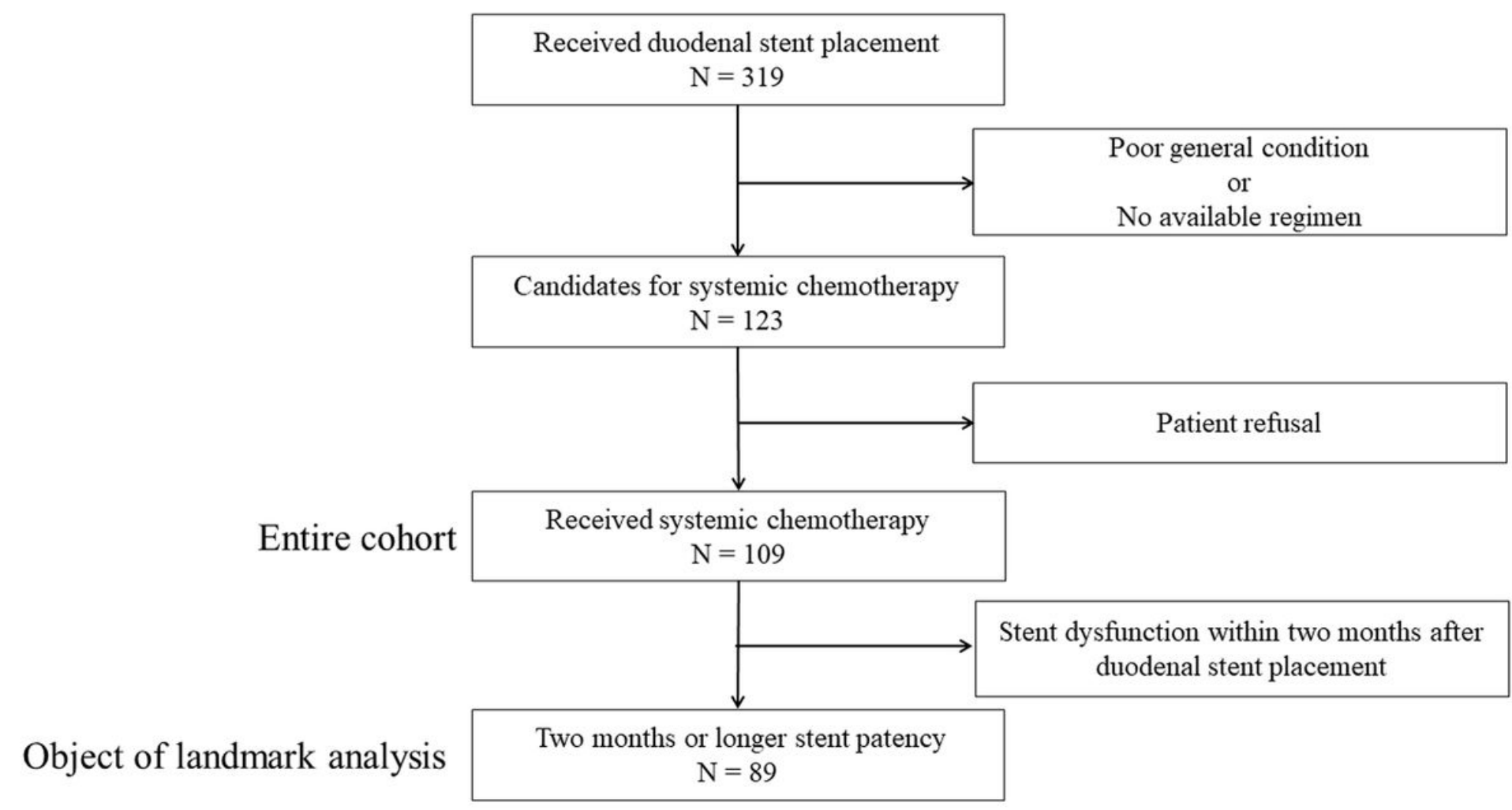

Figure 1

\section{Figure 1}

CONSORT flow diagram of the study Of 319 patients who received duodenal stent placement for malignant gastric outlet mobstruction due to advanced pancreaticobiliary cancer, 109 received systemic chemotherapy followed by duodenal stent placement, and 89 had two months or longer to stent dysfunction. 

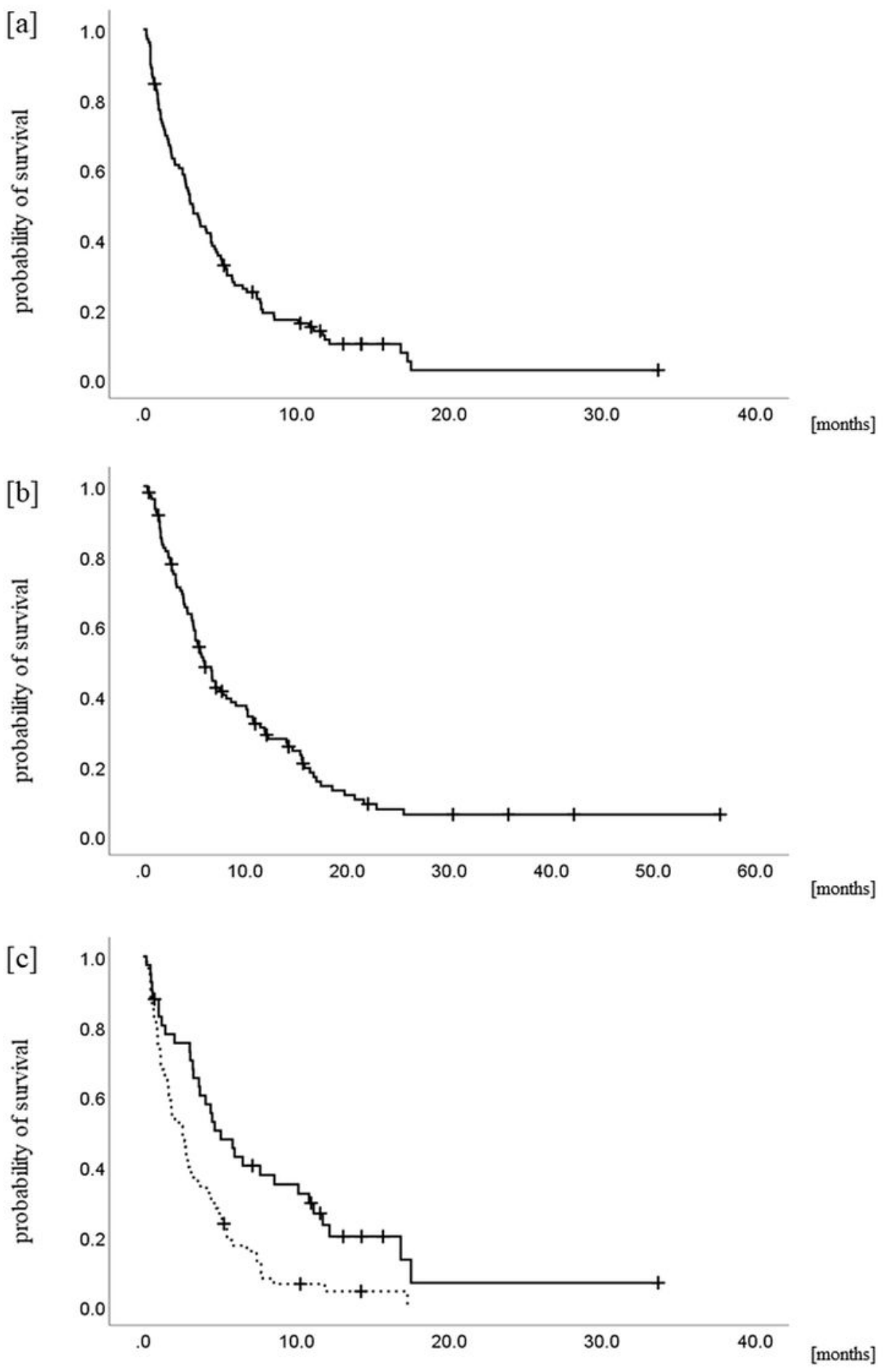

Figure 2

\section{Figure 2}

Kaplan-Meier curve of (a) overall survival and (b) progression-free survival In the entire cohort, which consisted of 109 patients, median progression-free survival and overall survival rates after duodenal stent placement were 3.2 months (95\% confidence interval, 2.4-4.0) and 6.0 months (95\% confidence interval, 4.6-7.3), respectively. (c) Comparison of 41 patients who received combination regimen (solid line) and 68 patients who received a monotherapy regimen (dotted line), median progression-free survival rates 
were 5.1 months (95\% confidence interval, 3.1-7.0) and 2.6 months (95\% confidence interval, 1.6-3.5) respectively, with a p-value of $<0.001$
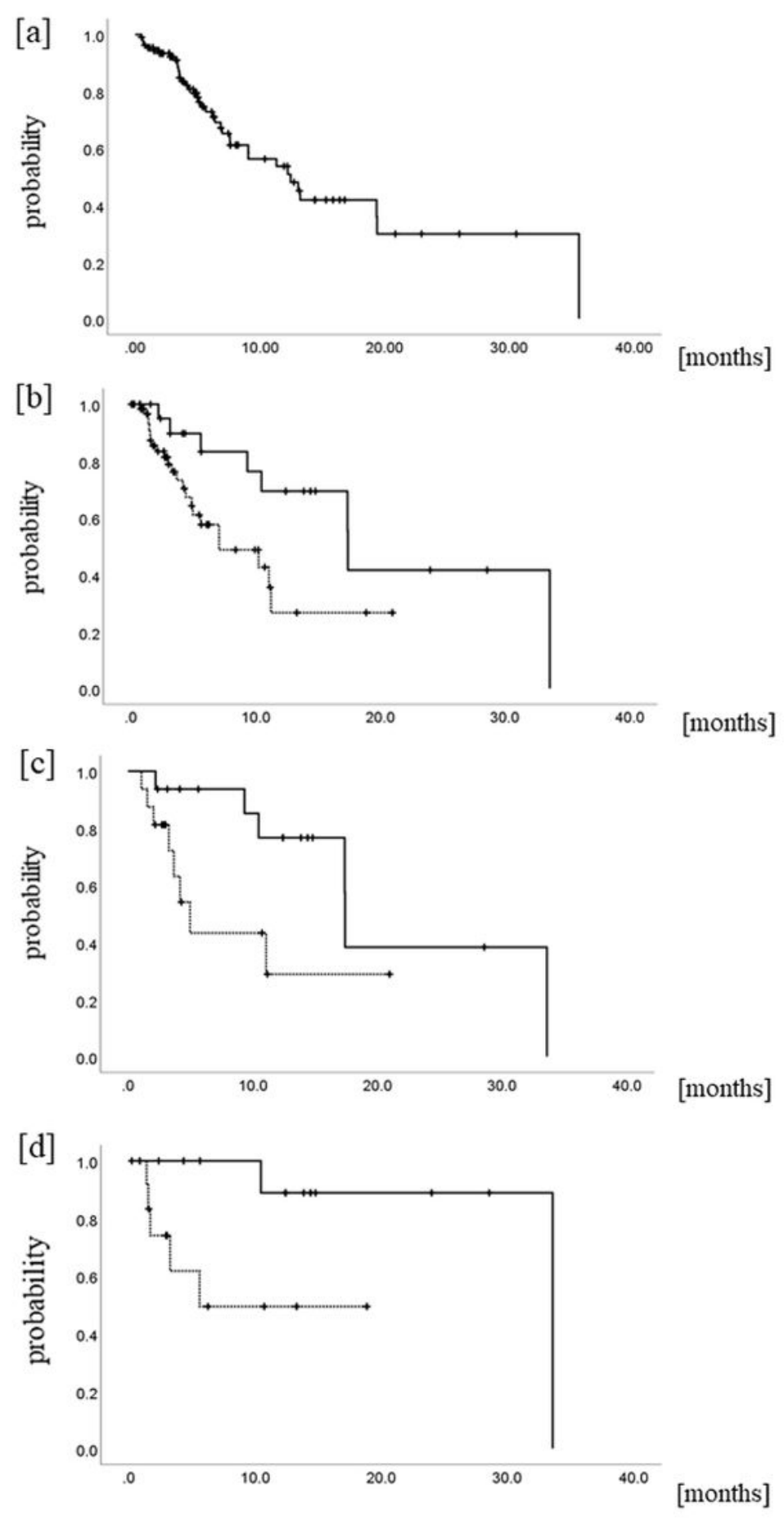

Figure 3

\section{Figure 3}

Time to stent dysfunction (a) Entire cohort Median time to stent dysfunction was 12.5 months (95\% confidence interval, 8.4-16.5); (b) Comparison between patients who showed a response (solid line) and those who did not (dotted line). Median time from the 2-month landmark point to stent dysfunction was 
17.4 months (95\% confidence interval, 17.3-17.5) in patients who showed a response and 7.1 months (95\% confidence interval, 1.6-12.5) in those who did not, with a p-value of 0.031 ; (c) Comparison between responders and non-responders among patients who received combination regimen. Median time from the 2-month landmark point to stent dysfunction was 17.4 months (95\% confidence interval, 10.2-17.5) in responders and 5.0 months (95\% confidence interval, 3.0-6.9) in non-responders, with a p-value of 0.043 ; (d) Comparison between responders and non-responders among patients who received systemic chemotherapy after duodenal stent placement as the first-line chemotherapy. Median time from the 2month landmark point to stent dysfunction was 33.6 months ( $95 \%$ confidence interval, non-evaluable) in responders and 5.6 months ( $95 \%$ confidence interval, non-evaluable) in non-responders, with a p-value of 0.024 .

\section{Supplementary Files}

This is a list of supplementary files associated with this preprint. Click to download.

- SuppleFigure1.tif

- SuppleFigure2.tif 